\title{
New algorithm for multi targets detection in clutter edge radar environments
}

\author{
Mustafa Subhi Kamal, Jiwa Abdullah \\ Communication Department, Faculty of Electrical Engineering, FKEE, University Ton Hussein on Malaysia (UTHM), \\ Malaysia
}

\begin{tabular}{l} 
Article Info \\
\hline Article history: \\
Received Jun 14, 2019 \\
Revised Sep 15, 2019 \\
Accepted Oct 6, 2019 \\
\hline
\end{tabular}

Keywords:

Algorithm

CFAR

Clutter

Multi-targets

Radar

\begin{abstract}
This paper deals with the problem of multi target detection that appears inside clutter cloud which represent the worst radar environments by using constant false alarm rate CFAR algorithm, in order to achieve maximum probability of detection with constant false alarm rate, to detect target in such environments it need to construct robust constant false alarm CFAR algorithm that excise the target spikes from CFAR window and deal with clutter edges in order to give best possible estimation to the noise background. Modified cell averaged (CA-CFAR) is analyzed and compared with Two important algorithms which are cell averaged (CA-CFAR) and ordered statistics (OS-CFAR) algorithms in additional to the modified CACFAR algorithm. All these algorithms were simulated with mat lab and applied them to matlab clutter test model that represent different radar environment cases. Tradeoff among these algorithms depending on their responses.
\end{abstract}

Copyright $(2020$ Institute of Advanced Engineering and Science. All rights reserved.

\section{Corresponding Author:}

Mustafa subhi Kamal,

Communication Department, Faculty of Electrical Engineering, FKEE,

University Ton Hussein on Malaysia (UTHM), Malaysia.

Email:ge170066@siswa.uthm.edu.my

\section{INTRODUCTION}

The performance of the radar receiver is greatly dependent on the presence of noise. Receiver has to achieve constant false alarm rate (CFAR) and maximum probability of target detection in order to detect. The signal returns from other electromagnetic reflecting sources referred to as interfering targets or unwanted echoes (clutter) from the ground, sea, rain or chaff and another electromagnetic wave reflecting source that interfere with the echo signal of the desired target. Since the clutter-plus-noise power not known at any given location, a fixed-threshold detection scheme cannot apply to detect the targets echo signal from the radar returns in individual range cells if the false alarm rate is to be controlled. An attractive class of schemes can used to overcome the problem of clutter and to maintain the constant false alarm rate (CFAR) processing schemes that set the threshold adaptively based on local information of total noise power.

Adaptive threshold technique based on the assumption that the probability density function of the noise known except for a few unknown parameters. The surrounding reference cells used to estimate the unknown parameters, and adaptive threshold value will be obtained. From experimental data, the clutter backscattering coefficient (effective echoing area) can be modelled the Rayleigh, exponential, the Weibull distribution or other depending on type of clutter. If the clutter returns are Raleigh envelope distributed, and they assumed identically distributed with the thermal noise, this constitutes the simplest clutter model. Sometimes the environment in which radar operates depends on factors that may yield statistically nonstationary signals with unknown variance at the receiver input. This non- homogeneous environment with clutter edge and non-uniform environments with unwanted multi interfering targets spikes need robust CFAR 
algorithm to deal with clutter edge and excise unwanted targets spikes from the background noise estimation to achieve primary wanted targets detection.

Many methods and algorithms used to deal with non-homogeneous and non-uniform environments, rohling designed ordered statistics (OS-CFAR) that considered novel idea and the beginning of many methods that based on that algorithm [1], such as trimmed mean (TM-CFAR)and censored mean level detector (CMLD-CFAR) [2]. After that new attitude to examine, the radar environment before applying suitable CFAR circuit established, and other attitude to use combination of CFAR methods together with selection criteria. to choose appropriate method according to the assumed environment such as variable indexed (VI-CFAR) [3]. Almost no CFAR algorithms dealt with multi target inside the clutter cloud. They did not propose this worst radar environments to happen, therefore it required robust algorithm to deal with this situation. It required very complex algorithm for the worst radar environment that need complex hardware with high processing time or may use two stage-censoring algorithm that make it impractical for real time applications. All of these algorithms may handle this situation with expense of hardware complexity that expense high processing time that make these algorithms unsuitable for radar real time applications.

New algorithm concept was necessary to overcome the high processing time problem and hardware complexity to use with radar real time applications. With a new idea that is suggested to excise the largest sample from leading and lagging accumulator (that summing leading and lagging samples)

\section{RESEARCH METHOD}

The main aim of this project is to construct new CFAR algorithm that can deal with clutter edge problem and able to solve multi-target problem. In addition, to evaluate this new suggested algorithm by constructing matlab test model to represent worst radar environment after simulated the proposed algorithm with matlab and apply it to matlab test model. Similarly, simulate the other CFAR famous algorithm in the procedures and apply to the same test model to trade off for choosing the algorithm that has the best response. Moreover, evaluate these algorithms depending on the processing time needed for radar real time applications. Construct mathematical modeling to the new designed algorithm.

\subsection{Test Protocol.}

Matlab testing procedures for proposed method as shown in Figure 1.

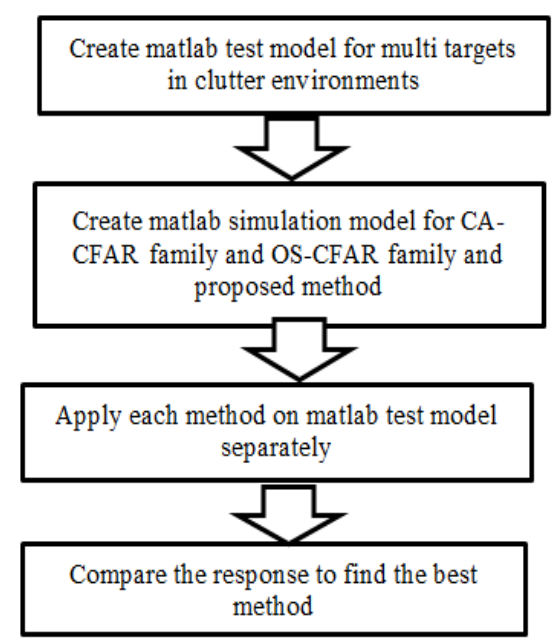

Figure 1. Matlab testing procedures for proposed method

\subsection{The CA-CFAR and OS-CFAR Algorithms}

The CA-CFAR family is shown in Figure 2. If the average of the surrounding reference cells used to estimate the background noise, then cell averaged CA-CFAR constructed, if the greater from leading reference window samples summation and the lagging reference window samples summation the greater of GO-CFAR constructed, and if the smaller between them selected the smaller of SO-CFAR constructed. The reference window constructed from one sample cells comes from analogue sampler for radar range echo signal transferred to digital code by analogue to digital convertor [3]. Ordered Statistics OS-CFAR algorithm 
is proposed for multi-target problems and need prior knowledge of the number of targets expected. The algorithm contains three steps the first step ranks order of the reference cells where the samples sorted in ascending order and in the second step it estimates the interference power as equal to the kth sample in ordered sequence. As $\mathrm{J}=$ predetermined number of targets and $\mathrm{M}=$ total number of reference cells that ranked according to their input level, then the only condition for OS-CFAR family to work properly is to choose the rank of the samples which their rank is less than (or equal) the result of subtracting the number of targets from the total number of reference cells $(\mathrm{K}=\mathrm{M}-\mathrm{J})$. Split sorting process for leading split of the reference window separately and lagging window as shown in Figure 3. And when the greater from leading and lagging selected the greater of ordered statistics GOOS-CFAR constructed, and when smaller selected smaller of ordered statistics SOOS-CFAR constructed $[1,4]$ as shown in Figure 4.

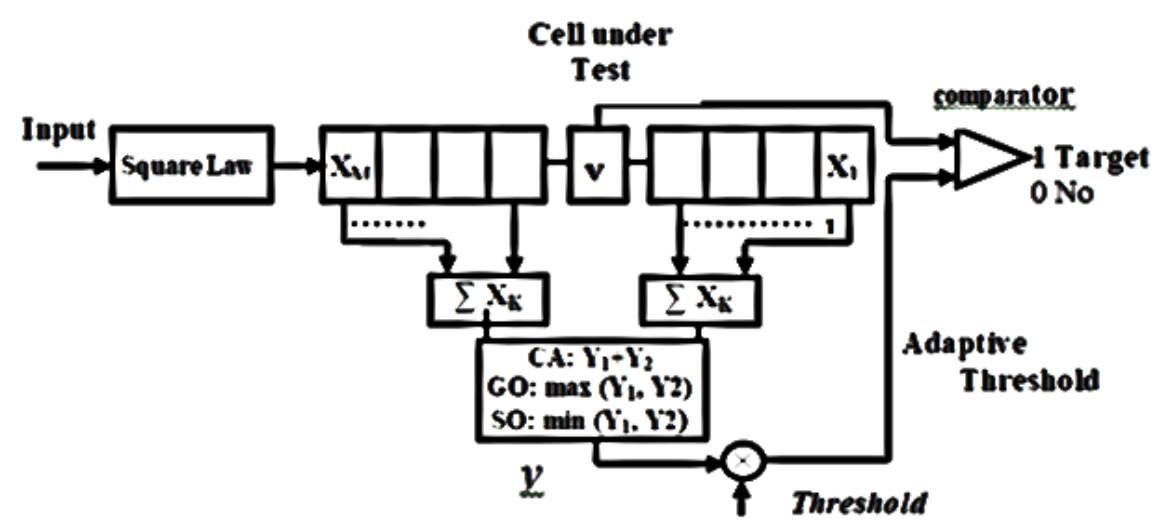

Figure 2. Block diagram of the CA-CFAR family processors

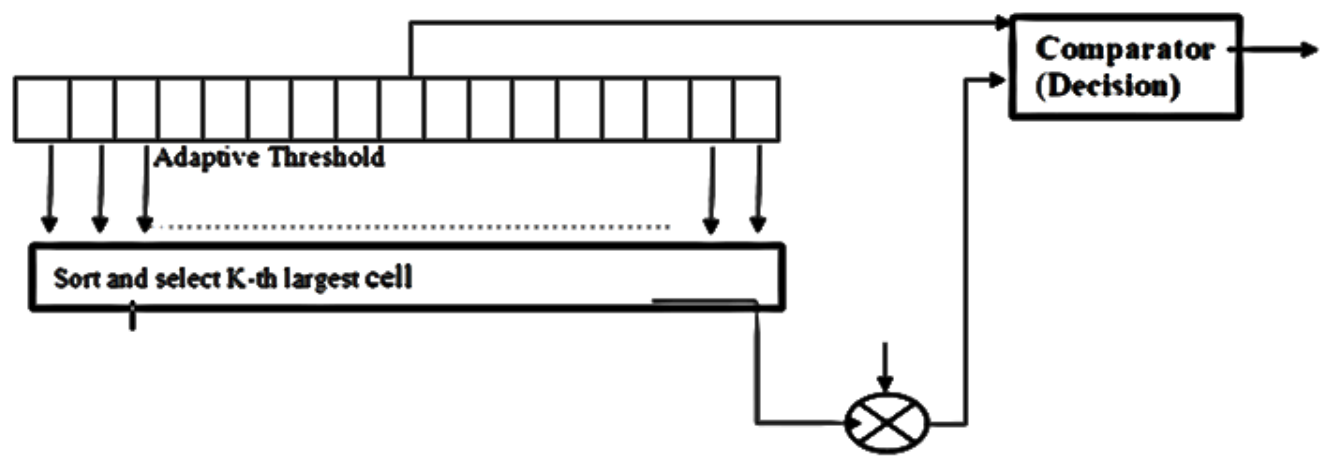

Figure 3. Block diagram of the OS-CFAR processor

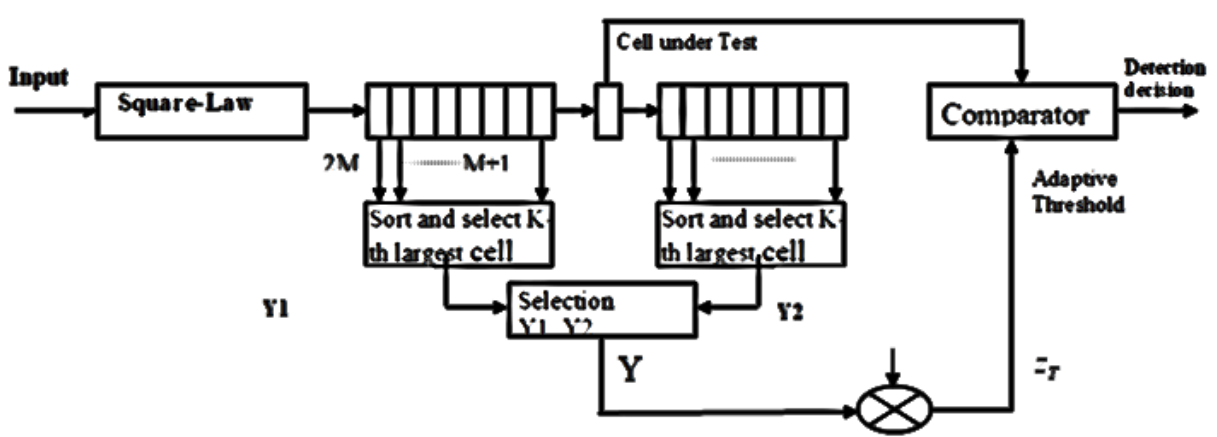

Figure 4. Block diagram of GOOS-CFAR and SOOS-CFAR processors 


\subsection{Construct New Algorithm}

The principle of this algorithm, modified CA-CFAR is to censor the strongest spike from leading reference cells and the strongest from lagging cells by using successive comparison technique that is not used before instead of rank and sort process in order to lessen processing time for radar real time applications that is need time reduction. The new algorithm constructed by three stages:

The first stage, serial input parallel output shift register is composed of $\mathrm{M}$ reference cells, which surround the cell under test located at the shift register centered tap. Range samples are sent serially into shift registers which contain $\mathrm{M} / 2$ leading reference cells and $\mathrm{M} / 2$ lagging reference cells and the cell under test. This window $(\mathrm{M}+1)$ moves along the time duration between two pulses until the entire cell tested.

The second stage, is two parallel processing circuits, the first is two accumulator summing circuits for the leading and the lagging windows. The second circuit is the two maximum sample lock circuits in the leading and the lagging windows. The maximum sample selected from leading and lagging window will be subtracted from the sum of samples in each half of the window.

The third stage, the result averaged and go the selection stage that select either the mean of the leading plus lagging window to construct the modified CA-CFAR, or select minimum or maximum of the leading and lagging to construct the modified SO-CFAR and the modified GO-CFAR respectively. As shown in the following Figure 5.

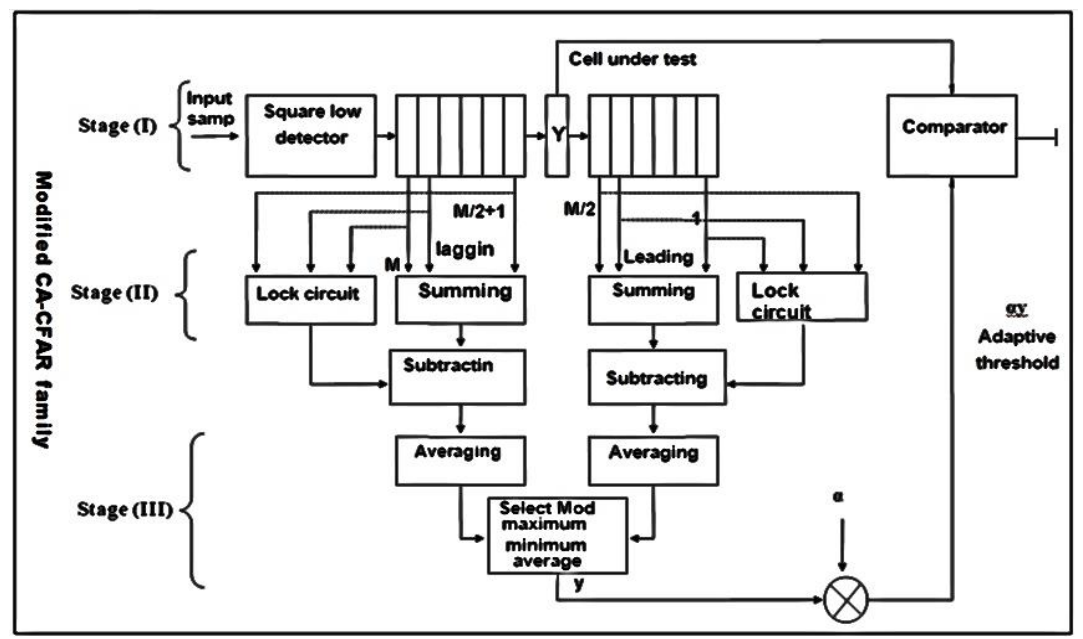

Figure 5. modified CA-CFAR family

\subsection{Essential Factors in Simulation Process}

To test these methods, radar environments will be created with models and each algorithm is applied separately to these models to examine the behavior of each of them in the worst radar environments and other environments. There are two factors of special importance when dealing with CFAR-algorithms which are the Size of Window (M) and closely separated targets. When M increased, the CFAR loss in a stationary noise background monotonically decreases, together with an increased hardware complexity. Also, with increasing in $\mathrm{M}$ an inevitable violation will occur of the inherent assumption that the noise samples are identically distributed over the reference window which is used to estimate the noise in the cell under test. Therefore, in a nonhomogeneous environment CFAR penalty sometimes increases with larger for large M, Also, the likelihood that an interfering target or a 'spiky' clutter return has entered the reference window is obviously larger for larger M [4, 7]. And in closely separated target's situation, another interfering target lies within the reference cells with the primary target (concerned target) the threshold is raised and detection of the primary target is seriously degraded. Sometimes closely separated targets called dense target environment, for example for a radar with a compressed pulse width of 1us and CFAR window with 16-cells on each side of the test cell and if two targets are within one antenna beam width and are separated in range less than $100 \mathrm{~m}$ the described suppression effect occurs. When clutter cloud appears within reference window the threshold value will rise and the probability of detection degraded, the larger length of clutter cloud (L) means more cell in the clutter cloud [5], and if clutter cloud with high power that give the probability of some small targets to immerse in that cloud. Also, if clutter cloud merged with multi targets the detection problem become more complicated, the worst radar environments when multi targets centered in clutter cloud [8-10]. 


\subsection{Weibull Probability Density Function}

CFAR procedures are originally developed using a statistical model of uniform background noise. However, this is not representative of real situations because it is impossible to describe all radar working conditions by a single model. For that reason, three different models with clutter clouds and stationary targets are chosen in different critical cases to make comparison between different CFAR procedures at the uniform clutter, clutter edges, and multiple target environments [11-13].

The entire 300 echo samples were generated by using Matlab from Weibull cumulative density function CDF that represented by (1).

If $\mathrm{x}$ is the amplitude of the output voltage, the Weibull CDF is.

$$
Q(x)=1-e^{\left(\frac{-x}{b}\right)^{a}}
$$

The Weibull probability density function PDF (which is the derivative of CDF)

$$
p(x)=\left(\frac{a}{b}\right)\left(\frac{x}{b}\right)^{a-1} e^{\left(\frac{-x}{b}\right)^{a}}
$$

These samples have Weibull PDF at constant skewness (shape) parameter (which is usually taken equal to 2), and it is known from (2) that with skewness (shape) parameter equal to 2 the Weibull PDF will take the form of Rayleigh PDF which is used in Matlab. Therefore, one can use Rayleigh distributed data for calculating multiplier factor to both OS-CFAR and CA-CFAR families [7, 14, 15]

The targets in these models are assumed to be Marcum targets (non-fluctuation) and the clutter cloud in each model always combines two clutter edges [16-18].

\subsection{The Testing Model}

The CFAR algorithm is tested by testing model that constructed from Multi targets that have different magnitudes and to make detection Procedure more complicated from the other models the multitarget are merged with clutter cloud and there is also closely separated targets which are located in different places. For more details there are $20 \mathrm{~dB}$ at 40 th and $50 \mathrm{~dB}$ at 50 th and $30 \mathrm{~dB}$ at 45th and 55th cell positions from 100th to 200th cell clutter cloud which is centered by five closely Targets $(2-30 \mathrm{~dB}, 2-40 \mathrm{~dB}, 70 \mathrm{~dB})$ respectively, and there is one target $40 \mathrm{~dB}$ Magnitude at 230th cell centered between two targets with $20 \mathrm{~dB}$ magnitude locations 225th and 235th cell respectively, as shown in Figure 6 [19, 20].

The CFAR algorithms deal with four situations, multi target, clutter cloud, multi target merged with clutter, multi-targets located inside clutter. In this model three regions shown: Closely separated multi targets merged with noise, multi targets cantered in clutter cloud representing the worst radar environments.

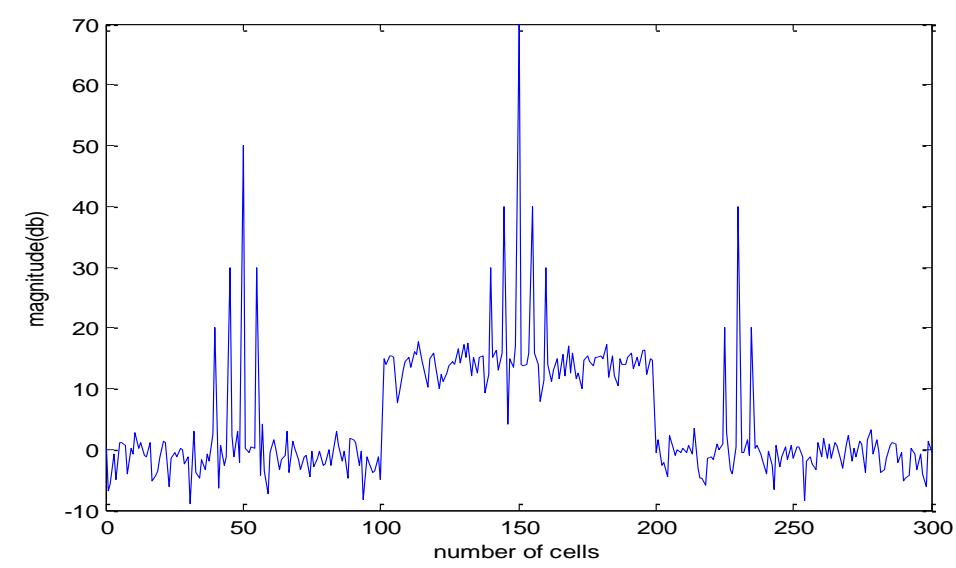

Figure 6. The test model

\subsection{Applying Matlab Simulated CFAR Algorithms}

The response of the CA-CFAR family to the test model as shown in Figure 7 and that family contained SO-CFAR and GO-CFAR, Figure 7 shows: GO-CFAR could only detect the strongest target, and fail to detect other targets.CA-CFAR also detects only the strongest target, and fails to detect other 
targets and also shows bad responses to the clutter edge. SO-CFAR detects all targets, but fall inside the clutter cloud.

OS-CFAR family give better response as shown in Figure 8:The targets are detected successfully, but the leading and falling clutter edge is missed by OSSO-CFAR while OS-CFAR misses only the falling clutter edge. It is shown clearly that OSGO-CFAR detects all the targets and did not affect with the Clutter edge problem. The output of OSGO-CFAR is larger than OSSO-CFAR and OS-CFAR which means a larger loss in $\mathrm{S} / \mathrm{N}$ ratio. The response of the OS-CFAR family to the test model and shows some progress from the response of CA-CFAR family, especially OSGO-CFAR that shows good response but with great loss. But it needed very high processing time because of sorting process of samples. On the other hand, from practical circuit design view OSGO-CFAR needs very high processing time and because of sorting process for the samples, it is very hard to implement in an electronic circuit. The OS-CFAR family takes larger window size $(\mathrm{M}=48)$, making the difficulties in background estimation, the only condition for OS-CFAR family to work properly is to choose the rank of the samples which their rank is less than (or equal) the result of subtracting the number of targets from the total number of reference cells, that means he number of the targets must be known to give a good estimation to the background noise.

The response of modified CA-CFAR more stable when compare with the other two families since it can detect small targets and detect the two edge of clutter cloud successfully and has the perfect response to noise and less probability of false alarm with less complicated algorithm without sorting process and therefore less processing time and hardware. The modified CA-CFAR, the new algorithm suggested is applied to the testing model as shown in Figure 9

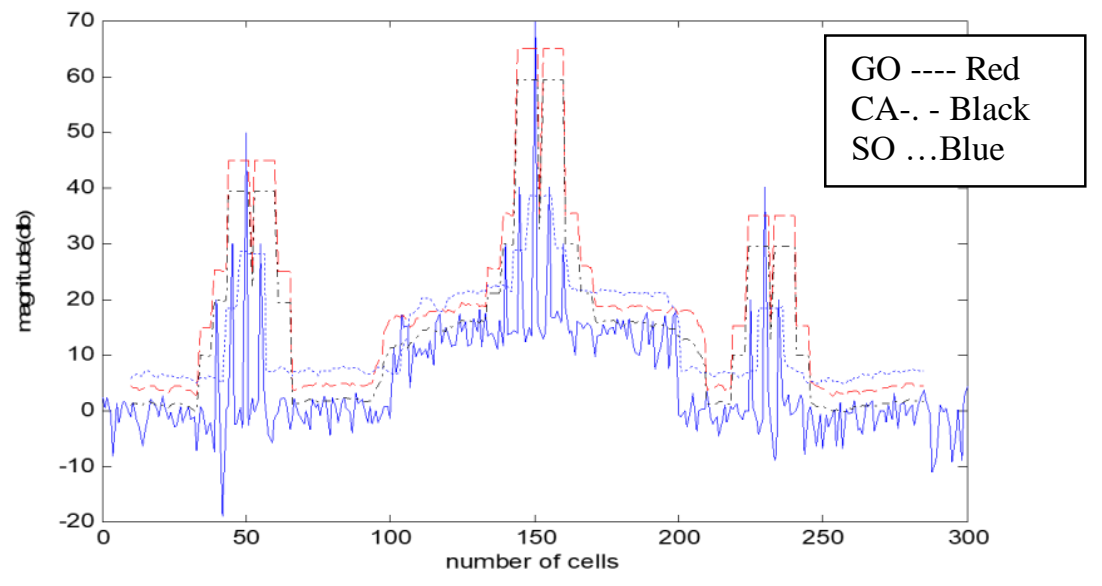

Figure 7. CA-CFAR Family with $\mathrm{M}=16$, applied to test model three $\mathrm{P}_{\mathrm{fa}}=10^{-6}$

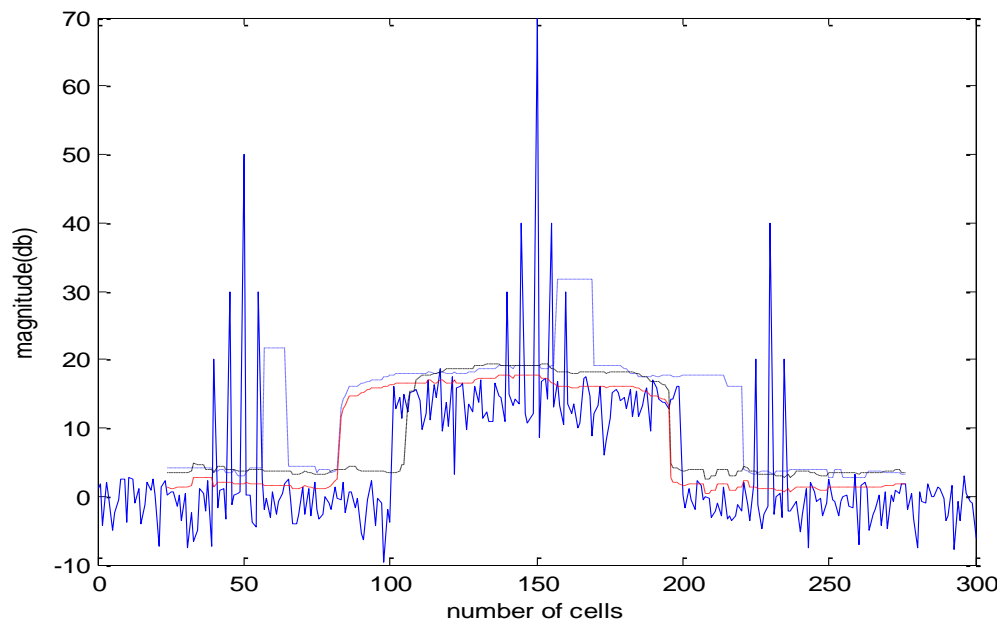

Figure 8. OS-CFAR family applied to test model, $\mathrm{M}=48, \mathrm{P}_{\mathrm{fa}}=10^{-6}$ 


\section{RESULTS AND ANALYSIS}

When applying CA-CFAR algorithm to the test model it will see from the Figure 7 that from CACFAR family only GO-CFAR can handle clutter edge in the model one and also in model two, while failing to handle clutter edge in test model.The OS-CFAR family has applied to the testing model as shown in figure (8) it behaved much better the CA-CFAR family in clutter edge and multi-target environment from especially OSGO-CFAR. OSGO-CFAR needs very high processing time and because of sorting process for the samples it is very hard to implement in an electronic circuit. Even OSGO-CFAR that needed only half processing time of OS-CFAR, the same hardware complexity of OS-CFAR sorting circuit is used. The modified CACFAR, the new algorithm suggested is applied to the testing model as shown in Figure 9, the proposed method showed superior response from other method especially in dealing with detecting multi targets centered in clutter cloud

\section{CONCLUSION}

Almost all the CFAR algorithms didn't deal with multi target inside the clutter cloud. They did not propose this worst radar environments to happen, their algorithms yield good performance in clutter edge or multi target, but it required robust algorithm to deal with this situation. The most probable algorithm that may deal with this condition is CFAR method that uses two censoring stage first stages censor interfering targets and the second stage deal with clutter cloud. It assumes that multi target is not centered in clutter cloud so it may handle the problem if multi target merged with clutter (but not inside clutter) and may handle this situation with the expense of hardware complexity that take very high processing time which is unsuitable for radar real time applications.

In order to solve the problem of target detection centered in clutter cloud which represent the worst radar environments, the algorithm of modified CA-CFAR will improve the performance of the airplane and ship navigation radar system. The algorithm could be used for preventing car collision system and avoid dangerous accidents with the real time CFAR algorithm. The algorithm could be used in the radar systems for highways recognition and limiting vehicle speed.

\section{ACKNOWLEDGEMENTS}

I would like to thank University Ton Hussein on Malaysia (UTHM), Malaysia. Communication Department, Faculty of Electrical Engineering, FKEE for their support and help.

\section{REFERENCES}

[1] H. Rohling. "Radar CFAR thresholding in clutter and multiple targetSituations". IEEE Transactions on Aerospace \& Electronics Systems, 19:608-621, July, 1983.

[2] WU. Mengjiao , J.YANG, C. ZHAO, X. HUANG , "The Design of Constant False Alarm Module Based on CMLD-CFAR algorithm". Department of Computer science and technology, Yunnan University, Kun Ming, 650500, China 4th International Conference on Machinery, Materials and Computing Technology (ICMMCT 2016.

[3] M.I.Skolnik., "Introduction to Radar Systems", 3d ed., McGraw-Hill, New York, 2001.

[4] A.R.Elias., M.G.Fuste de Marcado,., and E.R., Davo, "Analysis of Some Modified Order Statistic CFAR: OSGO and OSSO-CFAR”,. IEEE Trans.on AES-26,pp. 197-202,Jan.1990.

[5] p.p. Gandahi, and S. A. Kassam,. "Analysis of CFAR Processor in Non-Homogeneous Background”, IEEE Trans. on, AES-24 pp. 427-445, July 1988.

[6] L. Nadav "Radar Principles", John Wiley and Sons, USA, 1988.

[7] M.S. Kamal," Multi-Target. Detection in Cutter-Edge environments using modified GOCFAR". International Journal of Scientific \& Engineering Research, Volume 7, Issue 12, December-2016.

[8] F.D. Almeida Garcia ,A.C. Flores Rodriguez, "CA-CFAR detection performance in homogeneous Weibull clutter" IEEE Geoscience and remote sensing letter,29 November 2018.

[9] M. I. Skolnik ,"RADAR HANDBOOK”, McGraw-Hill Book Company, New York, Second edition, 1990.

[10] L.A Prastitis, ,'On adaptive censored CFAR detection'. Ph.D. thesis New Jersey Institute of Technology, January, 1993.

[11] A.Yousaf,M. Baig, ”Analysis of Cfar Techniques“, IEEE (IBCAST) conference islamabad pakistan2016.

[12] Y. Zhang, M. Gao, Y.Li ,"Performance analysis of typical mean-level CFAR detectors in the interfering target background", IEEE 9th Conference on Industrial Electronics and Applications(ICIEA), June 2014, China.

[13] M.A. Richards, J.A. Scheer, and W.A. Holm, Editors, Principles of Modern Radar: Basic Principles,SciTech Publishing, Raleigh, NC, 2010.

[14] Lv Dong, Zhang Yaotian, Yang Bin, Wang Jun, "The software pipeline realization of CFAR based on DSP” , IET International Radar Conference Oct.2015, China. 
[15] J. T Rickard and G.M.Dillard, "Adaptive Detection Algorithms for Multiple-Target Situations", IEEE Transactions on Aerospace and Electronic Systems, vol. AES-13, no. 4,July 1977.

[16] A.Abbadi , A. Abbane, M. L. Bencheikh, F. Soltani, "A New adaptive CFAR Processor in Multiple Target Situations", IEEE Seminar on Detection Systems Architectures and Technologies (DAT),Feb2017, Algeria.

[17] V.G. Hansen., "Constant False Alarm Rate Processing in Search Radar" Proceeding of the IEEE International Radar Conference, London, pp. 325-332, 1973.

[18] J.D.Moore, N.B. Lawrence, "Comparison of Two CFAR Methods Used with Square Law Detection of Swerling 1 Targets". Proceeding of the IEEE International Radar conference, 1980, 403-409.

[19] G.V Trunk, "Range Resolution of Targets Using Automatic Detectors", IEEE Trans. on , AES-14, pp. 750-755, September 1978. vol.18, No.1, pp. 102-113, January 1982.

[20] E. P. Blasch, M. Hensel ,'Fusion of Distributions for Radar Clutter Modeling” Air Force Research Lab, 2241 Avionics Cir-2003.

[21] Santos ,Rene ,Cesar ,'A Hybrid Approach for Target Detection using CFAR Algorithm and Image Processing" Proceeding of Fifth Mexican International Conference in Computer Science, 2004 IEEE.

[22] A. Farina and F.A. studer, "A Review of CFAR Detection Techniques in Radar Systems", Microwave Journal, vol. 29, No.9, pp. 115-128, September ,1986.

[23] R. Rifkin, "Analysis of CFAR Performance in Weibull Clutter", IEEE Trans on AES., vol. 30, No.2, pp. 315-327, 1994.

[24] P.Weber and S. Haykin, "Ordered Statistic CFAR Processing for Two Parameter Distributions with Variable Skewness", IEEE Trans. on AES., vol. 21, No. 6, pp. 819-821, November 1985.

[25] J. A. Ritcey and J. L. Hines, "Performance of max-mean level detector with and without censoring", IEEE Trans. On AEROSPACE and electronic systems, VOL.AES-25, NO. 2 MARCH 1989.

[26] F. García,A. Rodriguez ,G Fraidenraich and J Filho. "CA-CFAR Detection Performance in Homogeneous Weibull Clutter“. IEEE Geoscience and Remote Sensing Letters, VOL. 16, NO. 6, JUNE 2019.

\section{BIOGRAPHIES OF AUTHORS}

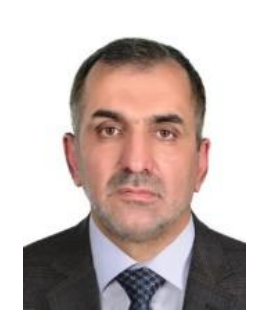

Mustafa subhi kamal

BSc. Mosul university 1990

Engineer in iraqi ministery of industry

MSc. Almustansiriya university

Researcher in ministry of scince and technology-iraq

Lecture in aliraqi university iraq

PHD. Student in university tun hussein on malaysia

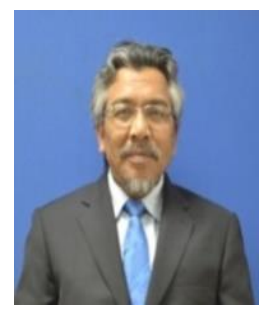

Assoc Prof. Dr Jiwa Abdullah received his Bachelor of Eng degree from Liverpool University, United Kingdom, in electronic engineering. He obtained his MSc degree and PhD from Loughborough University, UK. Currently he is attached to University Tun Hussein Onn Malaysia, Johor, Malaysia. His main interests are wireless sensor networks, underwater wireless sensor networks, mobile ad hoc networks, networking, application of computational intelligence to communication systems, wireless communications, integration of WSN and IoT and also in the area of engineering educations. 